\title{
What do we know about chronic renal failure in young adults? I. Primary renal disease
}

Guy H. Neild

Published online: 7 April 2009

(C) IPNA 2009

\section{Erratum to: Pediatr Nephrol}

DOI 10.1007/s00467-008-1108-3

Table 1 shows the epidemiology of the country regarding paediatric chronic renal failure. Unfortunately, there is a mistake relating to Turkey.

The online version of the original article can be found at http://dx.doi. org/10.1007/s00467-008-1108-3

G. H. Neild $(\bowtie)$

University College London (UCL) Centre for Nephrology,

Royal Free Campus,

London NW3 2QG, UK

e-mail: g.neild@ucl.ac.uk 
The correct Table 1 is given below.

Table 1 Epidemiology of paediatric chronic renal failure $(C R F)$ by country. Age is the age group in that registry. Country (CRF): most reports refer to patients starting dialysis. Some registries include all patients with chronic renal failure. CRF varies from $<30 \mathrm{ml} / \mathrm{min}$ per $1.73 \mathrm{~m}^{2}$ body surface area to $<75 \mathrm{ml} / \mathrm{min}$ per $1.73 \mathrm{~m}^{2}$ body surface area. Congenital is the percentage of patients with CAKUT, + hereditary is the sum of CAKUT plus hereditary disease. Missing values indicate data not available. NAPRTCS North American Pediatric Renal Transplant Cooperative, EDTA European Dialysis and Transplant Association

\begin{tabular}{|c|c|c|c|c|c|c|}
\hline Year of publication & Reference & Age (years) & Country & Number & Percentage Male & Congenital/+ hereditary \\
\hline 2007 & {$[1]$} & $<21$ & NAPRTCS (CRF) & 6794 & $64 \%$ & $51 / 61 \%$ \\
\hline 2006 & {$[2]$} & $<22$ & Poland & 469 & $56 \%$ & $/ 56 \%$ \\
\hline 2005 & {$[3]$} & $<16$ & UK & 845 & $61 \%$ & $47 / 67 \%$ \\
\hline 2005 & {$[4]$} & $<15$ & Kuwait & 171 & $73 \%$ & $67 / 88 \%$ \\
\hline 2005 & {$[5]$} & $<16$ & Holland & 351 & $56 \%$ & $38 / 62 \%$ \\
\hline 2005 & {$[6]$} & $<15$ & Bangladesh (CRF) & 44 & $68 \%$ & $60 / 67 \%$ \\
\hline 2004 & {$[7]$} & $<14$ & China (CRF) & 1268 & $60 \%$ & $/ 25 \%$ \\
\hline 2003 & {$[8]$} & $<20$ & Italy (CRF) & 1197 & $67 \%$ & $61 / 80 \%$ \\
\hline 2003 & {$[9]$} & $<19$ & Serbia (CRF) & 48 & $71 \%$ & $58 / 75 \%$ \\
\hline 2003 & {$[10]$} & $<18$ & India (CRF) & 305 & $74 \%$ & $58 / 66 \%$ \\
\hline 2003 & {$[11]$} & $<16$ & Nigeria (CRF) & 45 & $62 \%$ & $31 / 31 \%$ \\
\hline 2003 & {$[12]$} & & Pakistan & 78 & $65 \%$ & $47 / 61 \%$ \\
\hline 2002 & {$[13]$} & $<20$ & Japan & 582 & $57 \%$ & $38 / 57 \%$ \\
\hline 2002 & {$[14]$} & $<13$ & Jordan & 202 & $56 \%$ & $47 / 77 \%$ \\
\hline 2002 & {$[15]$} & $<12$ & Jamaica & 34 & $62 \%$ & $41 \% /$ \\
\hline 2001 & {$[16]$} & $<16$ & Iran (CRF) & 166 & $57 \%$ & $54 / 75 \%$ \\
\hline 1999 & {$[17]$} & $<18$ & Chile & 227 & $51 \%$ & $56 / 67 \%$ \\
\hline 1998 & {$[18]$} & $<21$ & NAPRTCS (CRF) & 1725 & $67 \%$ & $60 / 64 \%$ \\
\hline 1997 & {$[19]$} & $<16$ & Sweden (CRF) & 118 & $61 \%$ & $41 / 68 \%$ \\
\hline 1995 & {$[20]$} & $<16$ & Turkey & 459 & $55 \%$ & $55 / 67 \%$ \\
\hline 1994 & {$[21]$} & $<16$ & France & 127 & $57 \%$ & $/ 69 \%$ \\
\hline 1990 & {$[22]$} & & Saudi Arabia & 100 & & $51 / 69 \%$ \\
\hline 1985 & {$[23]$} & $<16$ & Germany & 623 & & $45 / 64 \%$ \\
\hline 1980 & {$[24]$} & $<18$ & Miami, USA & 81 & & $57 / 59 \%$ \\
\hline 2004 & {$[25]$} & $<19$ & EDTA, EU & 3184 & & $32 / 50 \%$ \\
\hline 1999 & {$[26]$} & $<20$ & USRDS, USA & 4989 & $57 \%$ & $21 / 37 \%$ \\
\hline
\end{tabular}

